\title{
Proyecto CRASH: enseñando cinemática y dinámica en el contexto del análisis pericial de accidentes
}

\author{
Jordi Domènech-Casal ${ }^{1,2, a}$, Jesús Gasco ${ }^{1, b}$, Pere Royo $^{3, c}$, Santi Vilches ${ }^{4, \mathrm{~d}}$ \\ ${ }^{1}$ Institut Marta Estrada. Granollers (Barcelona). España. \\ ${ }^{2}$ Departamento de Didáctica de las Matemáticas y las Ciencias Experimentales. Universitat Autònoma de \\ Barcelona (Barcelona). España. \\ ${ }^{3}$ Institut-Escola Les Vinyes. Castellbisbal (Barcelona). España. \\ ${ }^{4}$ Institut Vilamajor. Sant Pere de Vilamajor (Barcelona). España. \\ -ajdomen44@xtec.cat,-, jgasco@xtec.cat, ${ }^{\mathrm{c}}$ proyo4@xtec.cat, ${ }^{\mathrm{d}}$ svilches@xtec.cat
}

[Recibido: 18 Septiembre 2017. Revisado: 30 Noviembre 2017. Aceptado: 23 Diciembre 2017]

\begin{abstract}
Resumen: Las metodologías activas, como la indagación y el aprendizaje basado en proyectos, se han propuesto como métodos para el desarrollo de la competencia científica. El método del estudio de casos es un tipo concreto de aprendizaje basado en proyectos que incorpora procedimientos de indagación y puede ser de utilidad para el desarrollo de las dimensiones conceptual, procedimental y epistémica de la competencia científica. Se presenta una experiencia inicial de desarrollo de actividades de estudio de caso para el aprendizaje de la cinemática y la dinámica en el contexto del peritaje de accidentes de tráfico. Se evalúa su impacto en el desarrollo de conceptos y procedimientos científicos y se discuten vías y herramientas para este tipo de metodología.
\end{abstract}

Palabras clave: Estudio de caso; Cinemática; Dinámica; Aprendizaje basado en proyectos; Competencia científica.

CRASH: a project to learn kinematics and dynamics in the context of court evaluation of traffic accidents

Abstract: Inquiry and Project-Based Learning methodologies have been proposed as methods to develop scientific competence. The Case Studies method is a kind of Project-Based Learning that incorporates inquiry procedures and can be useful for developing conceptual, procedural and epistemic dimensions of scientific competence. We present a learning experience on Kinematics and Dynamics based on Case Studies contextualized in traffic accidents evaluation by court experts. We assess its impact on the development of scientific concepts and procedures and we discuss tools and didactic strategies for this methodology.

Keywords: Case Studies; Kinematics; Dynamics; Project-Based Learning; Scientific competence.

Para citar este artículo: Domènech-Casal J., Gasco J., Royo P., Vilches S. (2018) Proyecto CRASH: enseñando cinemática y dinámica en el contexto del análisis pericial de accidentes. Revista Eureka sobre Enseñanza y Divulgación de las Ciencias 15 (2), 2103. doi: 10.25267/Rev_Eureka_ensen_divulg_cienc.2018.v15.i2.2103

\section{Introducción}

\section{La enseñanza de la cinemática y la dinámica en la Educación Secundaria}

En la enseñanza de los ámbitos de la Física de cinemática y dinámica, varios autores describen que algunas concepciones erróneas en física (como la visión de fuerza como «ímpetu» asociado al movimiento, o la visión del movimiento como un proceso en lugar de un estado, o la necesidad de fuerzas que existen de modo teleológico: "para que no se caiga») son muy resistentes al cambio y permanecen inalteradas por la enseñanza formal (Sebastià 1984, 1988). Incluso alumnos que inician estudios de ingeniería mantienen errores de concepto en lo relativo a las magnitudes de posición, velocidad y aceleración (Fuentes 2016). Añadidas a las ya mencionadas y con más énfasis en la Dinámica, otros autores (Mora y Herrera 2009) añaden concepciones como «los objetos permanecen en reposo a menos que una fuerza actúe sobre ellos», «los objetos inanimados no ejercen fuerza» o «una fuerza constante produce una velocidad constante».

\author{
Revista Eureka sobre Enseñanza y Divulgación de las Ciencias \\ Universidad de Cádir. APAC-Eureka. ISSN: 1697-011X \\ http://dx.doi.org/10.25267/Rev_Eureka_ensen_divulg_cienc.2018.v15.i2.2103 \\ http:/ / reuredc.uca.es
}


Algunos autores atribuyen el desarrollo de concepciones erróneas sobre física y, particularmente, relativas a la cinemática, al excesivo peso del trabajo de cálculo, algoritmos y estrategias estándar de resolución de problemas académicos (Alzugaray, Enrique y Esterkin 2014). Para afrontar esta situación, las orientaciones didácticas han incidido en dos aspectos: el papel del contexto vinculado a la esquematización gráfica y las propuestas investigativas.

En lo relativo al papel del contexto, varios autores defienden que el trabajo a partir de contextos relevantes como la seguridad vial u otros contextos cotidianos tienen gran utilidad en la modificación de concepciones previas sobre el movimiento y sus componentes (Sánchez et al. 1993, Guidugli, Fernández y Benegas 2004), en especial cuando se vinculan a su estudio gráfico. La vinculación de imágenes o vídeos de contextos reales con los vectores que representan las magnitudes físicas que participan en ellos ha sido propuesta como una vía de trabajo (Mendoza, Ripoll y Ruz 2005), también mediante el análisis con programas informáticos y sensores (Ezquerra, Iturrioz y Díaz 2012, Hurovich, Azpiazu, Cucci y Joselevich 2015).

Por otro lado, varios autores recomiendan (Mora y Herrera 2009) que la enseñanza de la física debe orientarse a la resolución de problemas, proporcionando la mayor cantidad posible de experiencias en el marco de conflictos, que pueden enmarcarse en pequeños proyectos de investigación (Picquart 2008) que impliquen la discusión y razonamiento en equipo. En este ámbito, algunos autores proponen el uso de sensores (Hurovich et al. 2015), una posibilidad que se ha expandido con las prestaciones de los teléfonos móviles (cronómetros, acelerómetros, GPS...) (López 2015), las posibilidades de la programación y la robótica vinculada al movimiento (Simarro, López, Cornellà, Peracaula, Niell y Estebanell 2016) y el esfuerzo llevado a cabo por distintos proyectos de generación de materiales para el trabajo con sensores y simuladores, como el proyecto europeo Go Lab (http://www.go-lab-project.eu/). Para que el trabajo investigador y experimental conlleve cambio conceptual, varios autores insisten en la necesidad de incorporar la justificación, el debate y el meta-conocimiento en el aprendizaje de la física (Hewson y Beeth 1995) y advierten de las dificultades que algunas aproximaciones indagadoras presentan para el desarrollo de modelos científicos suficientemente completos y vinculados a otros modelos científicos (Viennot 2011).

En el currículo LOMCE español (MECD 2015), la cinemática y la dinámica se proponen como parte de los aprendizajes vinculados a la materia de Física y Química para $2^{\circ}$ y $3^{\circ}$ de ESO (en forma de criterios de evaluación): el reconocimiento de las fuerzas como causa de los cambios en el estado de movimiento y de las deformaciones, el concepto de velocidad como relación entre el espacio recorrido y el tiempo invertido en recorrerlo y el uso de gráficos para identificar la aceleración y distinguir entre velocidad media e instantánea. Los estándares que concretan estos criterios evitan en todo momento mencionar explícitamente el uso de fórmulas y priman una perspectiva cualitativa de las relaciones entre magnitudes físicas: «establece la relación» «deduce» «justifica» mencionando sólo en uno de ellos el cálculo cuantitativo de la velocidad. También priman el contexto y la interpretación de gráficos. Esto no solo converge con las recomendaciones de los autores mencionados anteriormente, sino que además es comprensible por el todavía básico conocimiento matemático del trabajo con ecuaciones que el mismo currículo propone para estos niveles educativos.

\section{Marco teórico}

\section{El desarrollo de la competencia científica}

El desarrollo de la competencia científica implica el uso de modelos científicos, pero también el desarrollo de habilidades y estrategias de construcción de conocimiento (Jiménez- 
Aleixandre 2011). La OCDE (2013) propone estructurar el desarrollo de la competencia científica en tres dimensiones, que pueden constituir un marco operativo para la evaluación y el diseño de actividades (Garrido y Simarro 2014). La dimensión conceptual comprende el dominio de conceptos y modelos científicos y su aplicación a contextos reales. La dimensión procedimental incluye las prácticas y habilidades de razonamiento científico en que se basa la investigación empírica. La dimensión epistémica trata el marco lógico y social en que se desarrollan las preguntas y teorías y la naturaleza de la ciencia. Si bien este marco de trabajo es una orientación útil para determinar qué aspectos deberíamos desarrollar para promover la competencia científica, el tipo de experiencias o actividades que llevan a ello es un tema más complejo.

\section{Metodologías hacia la competencia científica}

Los últimos años han adquirido una cierta efervescencia en la enseñanza de las ciencias dos bloques metodológicos: la Indagación y el Aprendizaje Basado en Proyectos. Los llamamos bloques porque en cada uno de ellos existen variantes con implicaciones en el desarrollo de la competencia científica.

La Indagación prima la idea que es necesario que los alumnos participen en trabajos prácticos de corte investigativo, en el que se pongan en juego las estrategias de creación y validación del conocimiento científico (Osborne y Dillon 2008). Si bien existen distintos enfoques de esta aproximación didáctica (Simarro, Couso y Pintó 2013), ésta suele identificarse con la Enseñanza de las Ciencias Basada en la Indagación (ECBI). La ECBI propone que el alumnado construya en primera persona los modelos que constituyen el conocimiento científico mediante la investigación científica (Llewellyn 2005, Rocard 2007). Por su potencial metodológico, la ECBI se ha promovido desde distintas instituciones internacionales (Rocard 2007). No obstante, varios autores advierten que esas expectativas no se han cumplido del todo (Hattie 2009), principalmente por dos razones: 1) es difícil que los alumnos consigan inducir por sí mismos y a partir de pequeñas investigaciones escolares modelos científicos complejos a un nivel suficiente como para formar un marco global (Viennot 2011), y 2) la ECBI en su aplicación ha desarrollado a menudo versiones pobres en eventos de modelización y argumentación (Couso 2014). La diversidad de aplicaciones y discursos alrededor de la Indagación ha hecho que sea difícil establecer de forma genérica una valoración sobre la aportación de esta aproximación al desarrollo de la competencia científica.

El Aprendizaje Basado en Proyectos (ABP) es una de las respuestas al requerimiento metodológico de un contexto como vía para generar aprendizajes profundos y transferibles (Gilbert 2006, Blanco, España y Rodríguez 2012, Sanmartí 2016, Sanmartí y Márquez 2017). Los cuatro tipos de Aprendizaje Basado en Proyectos propuestos inicialmente por Kilpatrick (1918) corresponden a distintos propósitos de la actividad escolar: elaborar un producto, disfrutar una experiencia estética, resolver un problema o desarrollar una habilidad o conocimiento concreto). Excepto el último, comparten la existencia de un objetivo externo al aprendizaje (construir un barco, hacer una reclamación al ayuntamiento), que llamaremos «objetivo del proyecto», una base que comparten las reformulaciones actuales de la metodología del ABP (Larmer, Mergendoller y Boss 2015, Domènech-Casal en edición). En su aplicación al diseño de actividades para la enseñanza de las ciencias, esto sitúa a los modelos científicos no como objetivo explícito de la actividad escolar (como ocurre en la Indagación), sino como instrumento para un objetivo externo (aprender flotabilidad para construir un barco, aprender el género lingüístico de la carta para hacer una propuesta al ayuntamiento). La presencia de un objetivo externo también provoca la conexión de la actividad escolar con contextos reales, dando lugar a escenarios en que el alumnado se autogestiona y planifica en distintos grados (actividades abiertas, estructuradas y cerradas) (Chiva y Martí 2016). Esta 
orientación a contextos reales suele provocar la inclusión de conocimientos de otras disciplinas, por lo que se acostumbra a relacionar el ABP con la interdisciplinariedad (Guillaumes 2016, del Moral 2016), algo que puede dificultar el desarrollo de dinámicas epistémicas propias de la Ciencia (Miró et al. 2016).

\section{Los estudios de caso}

La metodología de los estudios de caso es un tipo concreto de ABP que reúne también algunas dinámicas epistémicas propias de la ECBI. En ella, el punto de partida es un escenario que interpretar o resolver mediante la instrumentalización de los contenidos o procedimientos a aprender. Esta metodología es muy conocida en el ámbito universitario en la enseñanza de ciencias sociales (derecho, economía, administración de empresas...), y fue inicialmente aplicada en la Universidad de Harvard (Christensen y Hansen 1986). Valorada como metodología para un desarrollo más profundo de conocimientos y procedimientos (Hudson y Buckley 2004, Domènech-Casal 2017a), fue posteriormente exportada a la enseñanza de las ingenierías y las ciencias como la medicina (Hutchings 1993, Herreid 1994). Los casos pueden ser construidos y resueltos en forma de breve diálogo socrático de una sesión o con el apoyo de procesos de búsqueda, contrastación de información e indagación de más duración (Pollatsek y Schwartz 1990). Como puntos fundamentales en las secuencias de estudio de caso se proponen (Wasserman 1999):

-El punto de partida es una situación inicial contextualizada (real o verosímil). Los contextos o escenarios propuestos no es necesario que sean reales, pueden ser recreaciones verosímiles de la realidad, lo que permite el trabajo con escenarios ficticios, como por ejemplo el trabajo con secuencias didácticas de genética forense (Sebastiany et al 2013).

-La situación contiene un problema a resolver a partir de la interpretación y ponderación de pruebas.

- La resolución del caso implica el uso instrumental de modelos teóricos y dinámicas de discusión y argumentación.

El «método de caso dirigido» es un tipo concreto de estudios de caso (Cliff y Wright 1996, Cliff y Nesbitt-Curtin 2000), que, además, de las características generales para los estudios de caso, cumple las siguientes características:

-Después de proponer el caso inicial, se proporcionan al alumnado nuevas pruebas de forma paulatina (Glew 2003, Herreid 2003), emulando la forma en que en la Ciencia aparecen las evidencias y provocando cambios en las conclusiones.

-En paralelo a la resolución del problema, los alumnos reciben clases teóricas sobre los modelos científicos implicados para que establezcan conexiones entre los modelos científicos y el contexto en que se ubica el problema a resolver.

\section{Contexto, objetivos y metodología}

Consideramos que los estudios de caso son una metodología prometedora en lo que respecta al desarrollo de las tres dimensiones de la competencia científica. Los accidentes de tráfico constituyen escenarios de interés para su estudio desde la cinemática y la dinámica: en casos de accidentes graves o en accidentes en los que está en discusión la responsabilidad, se llega a un juicio para dictaminar responsabilidades.

En estos juicios, a menudo, participa la figura del perito, un experto que analiza el accidente desde un punto de vista científico, partiendo de evidencias (estado y posición de los vehículos, testimonios, marcas o residuos en la calzada) que contrasta, determinando el grado de 
certidumbre de sus conclusiones para que sean evaluadas por el juez. Es un contexto muy prometedor para el trabajo competencial de la cinemática y la dinámica desde una perspectiva conceptual (magnitudes, vectores, representación y estudio del movimiento y sus causas), procedimental (trabajo investigador a partir de medidas indirectas y experimentación) y epistémica (establecer niveles de certidumbre de conclusiones y métodos de validación del conocimiento).

En este artículo nos proponemos:

口Presentar el diseño de una propuesta de enseñanza de la cinemática y dinámica a través del método de caso dirigido.

口Analizar la validez de ese diseño para el aprendizaje y el desarrollo de la competencia científica

\section{Diseño de la secuencia didáctica de estudio de caso}

Se ha elaborado una secuencia didáctica titulada $C R A S H$ ! alrededor del peritaje de accidentes automovilísticos, como estudio de caso dirigido. En la secuencia (materiales disponibles ${ }^{1}$ ) se propone a los alumnos que participen como peritos en la resolución de tres casos de accidentes automovilísticos, simulaciones creadas ad hoc (figura 1). Para cada caso disponen de varios tipos de evidencias (testimonios de los conductores, posición de los coches después del accidente, masa de los coches, etc.).

Para afrontar la resolución de los casos, los alumnos trabajan en equipos de 3-4 alumnos a lo largo de varias sesiones, en las que se forman como peritos y usan distintas técnicas (simuladores virtuales, experimentos COD DE SIIISTRE: 030117-01 manipulativos con modelos de coche,...) $)\left.^{\text {Testimoni1: }}\right|^{\text {Conductor cotxe blau. }}$

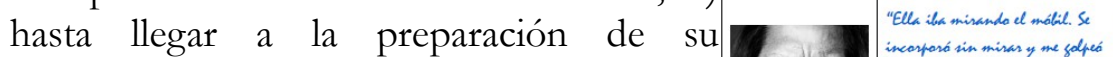
testimonio como expertos. Para el proceso se les ha ofrecido el apoyo de varios andamios lingüísticos (iniciadores de frase, conectores, léxico específico...).

En la tabla 1 se explicitan los objetivos de aprendizaje de la secuencia, en la que, de acuerdo con lo descrito en la introducción, se ha evitado centrar el trabajo en algoritmos y se ha promovido el trabajo en contexto y en base al análisis cualitativo, Figura 1. Parte de uno de los casos propuestos al alumnado. manipulativo y gráfico.

Tabla 1. Representación de las etapas de la secuencia. Cada etapa desarrolla un papel lógico en la narrativa (objetivo del proyecto) y promueve unos objetivos de competencia científica relacionados con las dimensiones conceptual (C), procedimental (P) y epistémica (E).

\begin{tabular}{|l|l|l|}
\hline Etapa y descripción & Objetivo en la secuencia & $\begin{array}{l}\text { Objetivo de aprendizaje y dimensión de } \\
\text { competencia científica asociada (C, P, } \\
\text { E) }\end{array}$ \\
\hline $\begin{array}{l}\text { 1.- Introducción y } \\
\text { motivación. }\end{array}$ & $\begin{array}{l}\text { Activar concepciones previas del } \\
\text { alumnado relativas a los accidentes de } \\
\text { Discusión sobre } \\
\text { accidentes que } \\
\text { conozico y aspectos epistémicos de su } \\
\text { sus causas y análisis de }\end{array}$ & $\begin{array}{l}\text { Identificar magnitudes físicas en el } \\
\text { contexto de los accidentes de tráfico. C } \\
\text { Comprender la importancia de la } \\
\text { comunicación científica. E }\end{array}$ \\
\hline \hline
\end{tabular}

${ }^{1}$ Descarga del dossier del alumno en https://app.box.com/s/nbndtbffmjh817iudvjyjriuoub3xxwz. 


\begin{tabular}{|c|c|c|}
\hline $\begin{array}{l}\text { agravantes. } \\
\text { Análisis de vídeos de } \\
\text { comunicación de } \\
\text { análisis periciales de } \\
\text { accidentes. } \\
\text { Trabajo guiado por el } \\
\text { profesor. Gran grupo. } \\
\text { 2h. }\end{array}$ & $\begin{array}{l}\text { peritos judiciales de accidentes de } \\
\text { tráfico. } \\
\text { Familiarizar al alumnado con formatos } \\
\text { de comunicación pericial. }\end{array}$ & \\
\hline $\begin{array}{l}\text { 2.- Análisis previo de } \\
\text { los casos. } \\
\text { Trabajo autónomo. } \\
\text { Equipos de trabajo. } 1 \mathrm{~h} \text {. }\end{array}$ & $\begin{array}{l}\text { Familiarizarse con los casos propuestos } \\
\text { y la estructura de la información. } \\
\text { Elaborar justificaciones previas para ser } \\
\text { contrastadas con los análisis finales. }\end{array}$ & $\begin{array}{l}\text { Desarrollar hipótesis y análisis de datos. P. } \\
\text { Activar léxico necesario relativo al tráfico } \\
\text { (encrucijada, ceda el paso,...) y evidencias } \\
\text { de accidentes (marcas de neumáticos, } \\
\text { desplazamiento,...) C. }\end{array}$ \\
\hline $\begin{array}{l}\text { 3.- Curso de } \\
\text { formación de peritos. } \\
\text { Los alumnos realizan } \\
\text { varias prácticas } \\
\text { relativas a la } \\
\text { medición, } \\
\text { representación y } \\
\text { cálculo de las } \\
\text { magnitudes asociadas } \\
\text { al movimiento. } \\
\text { Trabajo autónomo, con } \\
\text { breves explicaciones del } \\
\text { profesor. Equipos base- } \\
\text { gran grupo. 4h. }\end{array}$ & $\begin{array}{l}\text { Adquirir el léxico y conceptos clave } \\
\text { para poder analizar los casos desde una } \\
\text { perspectiva científica. } \\
\text { Familiarizarse con representaciones del } \\
\text { movimiento (vectores, gráficos) para } \\
\text { ser usadas en los análisis de casos. } \\
\text { Familiarizarse con los instrumentos } \\
\text { para el análisis de casos: rampas y } \\
\text { coches con masas variables y } \\
\text { laboratorio virtual simulador de } \\
\text { choques. }\end{array}$ & $\begin{array}{l}\text { Recolectar datos, representar gráficamente } \\
\text { la velocidad y el desplazamiento. Diseñar } \\
\text { experimentos, identificar correlaciones } \\
\text { entre variables y realizar predicciones a } \\
\text { partir de un gráfico. P } \\
\text { Argumentar a partir de datos usando } \\
\text { conectores gramaticales adecuados. P. } \\
\text { Conceptos de velocidad, aceleración, y su } \\
\text { relación con la fuerza. Concepto de línea } \\
\text { de tendencia, vector, fricción, pendiente, } \\
\text { distancia de frenada, masa y cantidad de } \\
\text { movimiento. C } \\
\text { Entender la media de un valor y el descarte } \\
\text { de datos desviados como aproximación } \\
\text { razonable. E }\end{array}$ \\
\hline $\begin{array}{l}\text { 4.- Análisis de los } \\
\text { casos. } \\
\text { Trabajo autónomo, con } \\
\text { breves explicaciones del } \\
\text { profesor. Equipos base- } \\
\text { gran grupo. } 5 \mathrm{~h}\end{array}$ & $\begin{array}{l}\text { Realizar análisis de los tres casos y } \\
\text { elaborar informes en los que se } \\
\text { presentan las evidencias consideradas } \\
\text { junto con el proceso seguido y las } \\
\text { conclusiones finales. }\end{array}$ & $\begin{array}{l}\text { Comunicar científicamente en un formato } \\
\text { científico profesional: el informe de perito. } \\
\text { P. } \\
\text { Entender las conclusiones científicas como } \\
\text { una explicación razonable a partir de las } \\
\text { evidencias, sujeta a grados de certidumbre } \\
\text { y consideraciones de ámbito social en su } \\
\text { aplicación (indemnizaciones, multas). E. }\end{array}$ \\
\hline $\begin{array}{l}\text { 5.- Audiencia. } \\
\text { Análisis de las } \\
\text { soluciones a los } \\
\text { casos. } \\
\text { Equipos base/gran } \\
\text { grupo. } 2 \text { h. }\end{array}$ & $\begin{array}{l}\text { Abrir la vista de cada uno de los casos, } \\
\text { y llamar a declarar a cada equipo sobre } \\
\text { dos de los tres casos analizados. } \\
\text { Actuar como abogados en el análisis } \\
\text { del trabajo de cada equipo de peritos. }\end{array}$ & $\begin{array}{l}\text { Escuchar argumentos y valorar su validez. } \\
\text { Valorar fuentes. P. } \\
\text { Conocer los rituales de comunicación } \\
\text { científica asociados al ámbito judicial } \\
\text { (protestas, relevancia, comprensibilidad). C, } \\
\text { P, E. } \\
\text { Entender la comunicación judicial de la } \\
\text { ciencia como un ámbito sujeto a códigos } \\
\text { no únicamente científicos. E. }\end{array}$ \\
\hline $\begin{array}{l}\text { 6.- Evaluación de la } \\
\text { actividad } \\
\text { Se realiza una prueba } \\
\text { orientada a las } \\
\text { dimensiones } C \text { y P de } \\
\text { la competencia } \\
\text { científica. } \\
\text { Individual. } 2 \text { h. }\end{array}$ & $\begin{array}{l}\text { Estructurar los aprendizajes, } \\
\text { Reflexionar sobre qué lo que se ha } \\
\text { aprendido e identificar dificultades. }\end{array}$ & $\begin{array}{l}\text { Sintetizar los conceptos base de cinemática } \\
\text { y dinámica. C. } \\
\text { Entender la necesidad de sistematizar } \\
\text { como modelos abstractos los } \\
\text { conocimientos científicos. E. }\end{array}$ \\
\hline
\end{tabular}


Tal como propone la metodología de estudios de caso dirigido, las sesiones prácticas (trabajo autónomo en equipos, orientado a la resolución del problema) se han alternado con intervenciones transmisoras en las que el profesor ha explicado y razonado elementos conceptuales (principios de dinámica newtoniana, representación de gráficos espacio/tiempo y vectores, concepto de fricción,...), en un modo similar al propuesto en ocasiones anteriores como protocolo TPoP (Domènech-Casal 2015). Las sesiones han sido de $2 \mathrm{~h}, \mathrm{y}$ al final de cada sesión los alumnos han realizado una autoevaluación de su trabajo y las ideas aportadas por cada miembro del equipo. Como se describe más adelante, en distintos puntos de la secuencia, los alumnos elaboran síntesis de conceptos clave mediante el modelo Frayer, un andamio de apoyo para el análisis y delimitación de conceptos (características principales, secundarias, ejemplos, no ejemplos...) (Frayer, Frederick y Klausmeier 1969).

\section{Diseño experimental para la evaluación de la secuencia}

La secuencia propuesta ha sido aplicada en distintos institutos de secundaria y se ha evaluado su aplicación en 66 alumnos de los institutos de secundaria Institut Marta Estrada (Granollers, Barcelona) e Institut-Escola les Vinyes (Castellbisbal, Barcelona). Los docentes participantes han recogido en forma de diario de aula distintas observaciones a lo largo de la aplicación de la secuencia.

Al final de la secuencia se ha llevado a cabo un examen ${ }^{2}$ en el que mediante 5 preguntas se han evaluado aspectos vinculados a las dimensiones conceptual y procedimental de la competencia científica, en concreto las capacidades de los alumnos para: 1) Representar y predecir cambios en el movimiento de los cuerpos; 2) Identificar modelos y magnitudes científicas en contextos concretos; 3) Definir conceptos y magnitudes físicas; 4) Representar y analizar movimientos 5) Interpretar representaciones gráficas para identificar los movimientos. Se ha calificado cada una de las preguntas sobre $10 \mathrm{y}$ se ha calculado la media obtenida por el total de alumnos para comparar entre sí los niveles de aprendizaje de cada uno de los ítems.

También al final de la actividad los 66 alumnos participantes han contestado a una encuesta en la que debían expresar su grado de acuerdo/desacuerdo mediante una escala Likert (1-6) a distintas frases relativas a qué aprendizajes consideran que han adquirido (conceptuales, procedimentales y epistémicos) y a qué elementos de la secuencia han sido de más utilidad para aprender. Los resultados de esta encuesta han sido resumidos como Apoya/no Apoya para facilitar el análisis. Como parte de esta encuesta, además, con el objetivo de evaluar el desarrollo de visiones epistémicas, una parte de los alumnos participantes (18 alumnos) ha respondido al inicio y final de la secuencia a 2 preguntas sobre el trabajo de los peritos: ¿Consideras que sus conclusiones son cientificas? ¿Consideras que son seguras? Los resultados se han resumido cuantitativamente como porcentaje de alumnos que apoya cada afirmación antes y después de la secuencia (Pre y Post).

Las aportaciones didácticas de la secuencia a las tres dimensiones de la competencia científica han sido analizadas mediante una roseta de dimensiones, tal como se propone en anteriores publicaciones (Domènech-Casal 2017a).

\section{Resultados sobre la aplicación de la actividad}

\section{Etapas 1 y 2. Activar concepciones previas y presentación del contexto}

En esta etapa, los alumnos han referido casos de accidentes conocidos por ellos y un breve análisis de sus causas. Si bien inicialmente se centran exclusivamente en aspectos como la atención o pericia de los conductores, en el debate se han hecho emerger magnitudes físicas:

\footnotetext{
${ }^{2}$ Examen y encuesta disponibles en https://app.box.com/s/735v8ds2mh9f6xo55qm6dzutwyv474ge
} 
en un día de lluvia el pavimento tiene menos fricción, un vehículo de gran masa es difícil de frenar...

Los alumnos realizan una interpretación inicial de los casos, para lo que se les proporcionan coches de juguete. $\mathrm{Al}$ discutir sus hipótesis sobre el posible desarrollo de los accidentes, los alumnos establecen espontáneamente la necesidad de un punto Figura 2. Alumnos elaborando sus hipótesis iniciales de referencia para el movimiento: «Esto es sobre el desarrollo de los accidentes.

la farola» «Aquí está el cruce» (figura 2), algo que se ha utilizado para discutir con ellos este concepto.

\section{Etapa 3. Desarrollo de conceptos y técnicas de representación y análisis}

En esta etapa los alumnos identifican las relaciones entre magnitudes (velocidad, aceleración, fuerza) y aprenden a reproducir, identificar y representar distintos tipos de movimientos (MRU, MRUA) con la ayuda de gráficos y vectores. Por un lado, los alumnos cronometran un desplazamiento de 20 metros en distintas circunstancias (caminando, corriendo, acelerando...) y representan y estudian el movimiento, identificando la pendiente como velocidad y trabajando las unidades de medida de la velocidad. Continúan además con el trabajo con modelos Frayer para definir cada nuevo

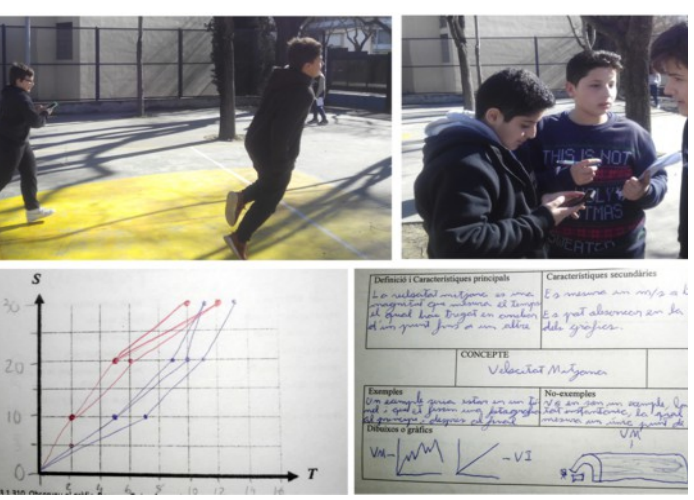

Figura 3. Alumnos realizando estudios sobre el movimiento cronometrando el desplazamiento de sus compañeros. concepto (Frayer et al. 1969) (figura 3).

Por otro lado, los alumnos realizan prácticas con el diseño experimental para desarrollar las relaciones cualitativas entre variables. Éste se compone de rampas de inclinación ajustable para dar distintas velocidades a coches de juguete, distintas superficies (papel, hule, cerámica) y lastres para cambiar la fricción y masa de los coches. Organizados en equipos, los alumnos logran establecer distintas relaciones (qué superficies tienen más fricción, qué efecto tiene la masa en el tiempo de frenada...) que comparten y presentan al resto del grupo (figura 4). Como síntesis, el profesor acompaña la formalización de los descubrimientos de los alumnos como las 3 leyes de la dinámica newtoniana (inercia, principio fundamental y acción-reacción) y el concepto de fricción. En esta etapa la mayor parte del alumnado ha sido capaz de diseñar experimentos con sentido, representar los datos y elaborar conclusiones a partir de ellos. Sólo dos equipos no han llegado a completar esta etapa por problemas de actitud y dispersión. En la conversación posterior, han emergido de forma repetida confusiones entre masa y peso, y aceleración y velocidad.

\section{Etapa 4. Análisis de los casos}

En esta etapa, los equipos de peritos empiezan a trabajar en los casos de forma autónoma (figura 5), teniendo a su disposición un simulador virtual de choques, las rampas y coches de juguete y las informaciones de cada caso. Los alumnos intentan determinar qué versión del accidente es pausible con los datos disponibles cambiando parámetros tentativamente de forma razonada en base a los modelos científicos. 

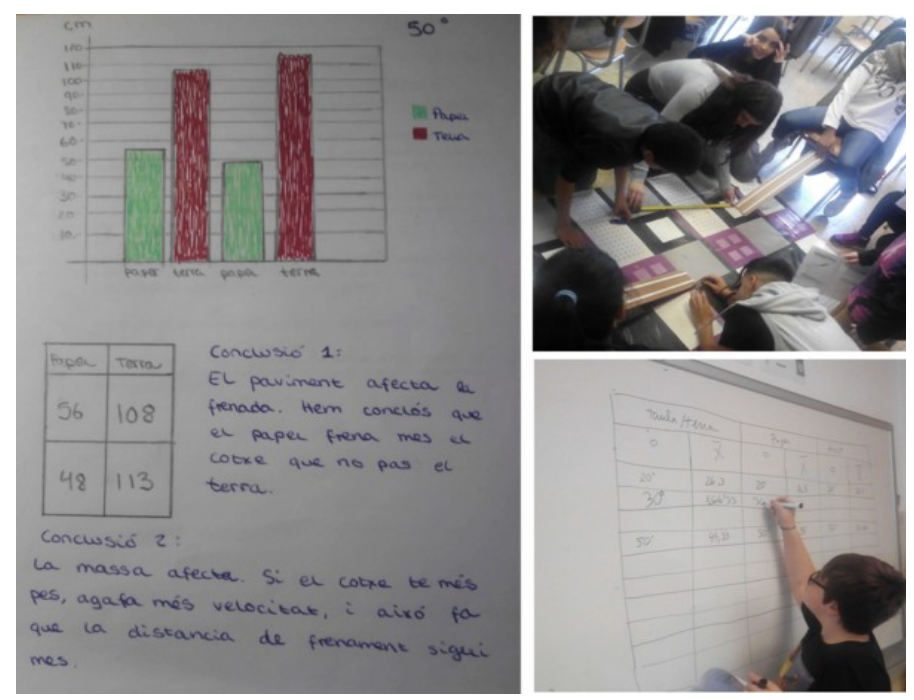

Figura 4. Alumnos determinando experimentalmente relaciones entre velocidad, distancia de frenada y fricción mediante distintos tipos de movimiento en el patio y en el uso de coches de juguete y rampas a distintas inclinaciones $y$ superficies.

\section{Etapas 5 y 6. Vista del juicio y evaluación}

Cada equipo de peritos prepara su exposición para cada caso, atendiendo a la presentación de evidencias, reconstrucción del accidente y determinación del grado de certidumbre. Este último dato es la expresión tentativa en porcentaje de cuán seguros están de sus conclusiones. Para agilizar y contextualizar el acto, el profesor ha actuado como juez llamando a declarar a 3 equipos de peritos para cada caso. Como dinámica epistémica, el profesor ha designado equipos de abogados defensores de cada conductor. Su misión ha sido velar por los intereses de su cliente, tal como establece el imaginario judicial televisivo, pudiendo interrumpir la declaración de un perito con la expresión «Protesto, señoría!». Los abogados han podido protestar cuando: 1) una conclusión no se basa en datos 2) no se determina correctamente el grado de certidumbre. Ante cada protesta, el profesor-juez escucha la protesta y la desestima (justificando por qué) o la acepta (solicitando a los peritos que declaran que corrijan su exposición in situ) (figura 6 y tabla 2). Las declaraciones de los peritos presentadas por los alumnos han hecho referencias a conceptos como inercia, masa, distancia de frenada, velocidad y términos como "posición inicial» o «posición final». En uno de los tres grupos clase la dinámica ha resultado negativa, pues algunos reconstrucción de uno de los accidentes incluyendo vectores de velocidad alumnos han acogido con y representación gráfica de la velocidad en los momentos anterior y incomodidad que otros posterior al momento del choque, identificado con una estrella en el compañeros les corrigieran en gráfico.

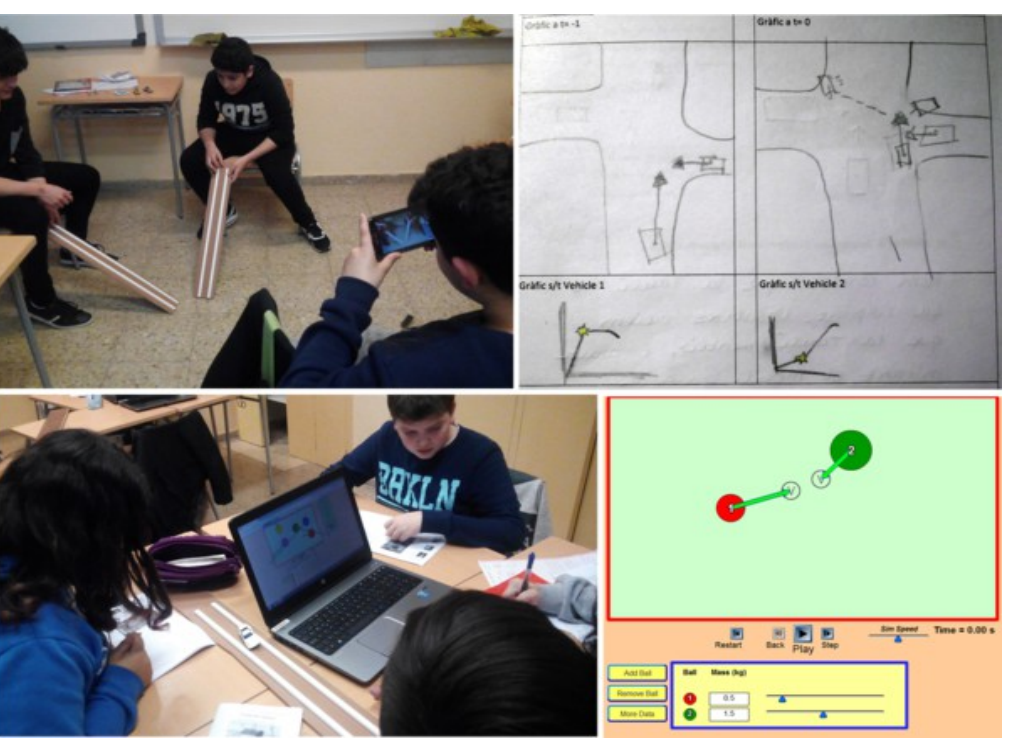

Figura 5. Equipos de peritos trabajando en la investigación de los casos y 
público.
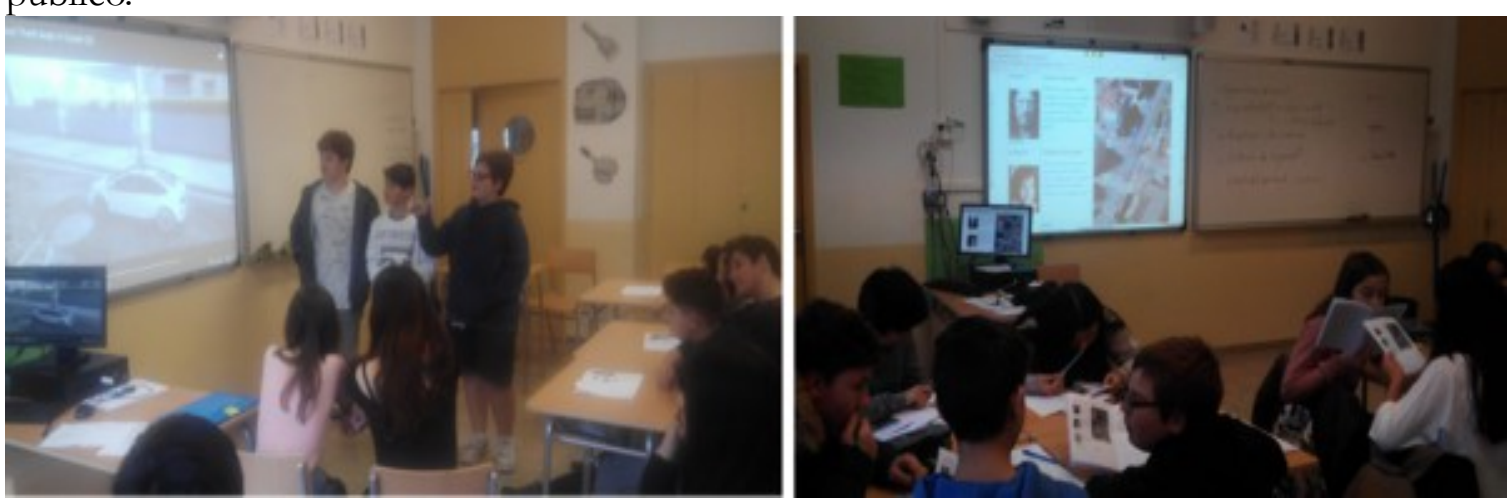

Figura 6. Momento de la vista del caso Sánchez contra Taulí en el que un equipo de tres peritos expone su reconstrucción del accidente bajo la supervisión de dos equipos de abogados.

Tabla 2. Reconstrucción de diálogo durante una vista del juicio de Sánchez contra Taulí.

Perito 1.- Y hemos conseguido hacer con las rampas el accidente como dice Sánchez, pero no como dice

Taulí. (muestra fotografía de coches de juguete después de chocar)

Perito 2.- Por eso pensamos que el accidente es como dice Sánchez.

Abogado 1.- ¡Protesto! No estáis diciendo si son conclusiones seguras.

Perito 2.- Ahora lo íbamos a decir...

Juez.- Denegada, dejad que hablen.

Perito 1.- Estamos bastante seguros, en un $70 \%$, porque en el simulador también sale sólo el de Sánchez. Hemos probado varias masas distintas, pero sólo se consigue si el coche de más masa (el de Sánchez) va más lento que el de menos.

Juez.- ¿....más lento...?

Perito 2.- A velocidad menor.

Perito 1.- Entonces, nos parece que los gráficos de velocidad de los dos coches serían estos (los muestra) y aquí es el punto donde chocaron (lo señala). Aquí se ve como después del choque los dos pierden velocidad (lo señala).
Perito 3.- Estamos bastante seguros de que Taulí iba más rápido -a más velocidad- que Sánchez. El coche de Taulí se movió más porque el de Sánchez es muy grande y por eso aunque vaya más lento empuja muy fuerte, le cuesta más de parar.

Juez.- ...es muy grande...y tiene más...

Perito 3.- Masa. Tiene más masa. Inercia, quiero decir inercia.

Abogado 2.- Pero no nos habéis enseñado las simulaciones.

Juez.- Aceptada la protesta. (a los peritos). Si sacáis conclusiones de unos datos tenéis que enseñarlos.

Perito 1.- Pero no los tenemos aquí.

Juez.- Se acepta la protesta. El hecho de no incluir los datos quita certidumbre a vuestro peritaje. Muchas gracias. Llamamos a declarar al equipo de peritos número 4.

\section{Análisis de los resultados del examen}

El examen realizado por los alumnos incluye distintas preguntas destinadas a evaluar aspectos conceptuales y procedimentales de la competencia científica. Las cinco preguntas evalúan distintos aspectos de competencia científica y (figura 7) observamos que las preguntas relativas a la dimensión procedimental obtienen de media mejores calificaciones que aquéllas relativas a la dimensión conceptual. De forma general, los alumnos hacen un buen uso de vectores y de los términos «dirección» y «sentido», y desarrollan aproximaciones rudimentarias de la conservación de la cantidad de movimiento al argumentar sobre trayectorias de choques entre objetos «Como el 1 tiene más velocidad, al nivelar la masa/velocidad el 2 aumenta la velocidad y el uno la disminuye», "Como más o menos pesan lo mismo al chocarse se igualaran las velocidades». En lo referente a las leyes de la dinámica los alumnos presentan en particular dificultades con el principio de acción-reacción. En cambio, desarrollan bien el principio fundamental de la dinámica, si bien con algunas confusiones en algunos casos identificando inercia con ímpetu. 


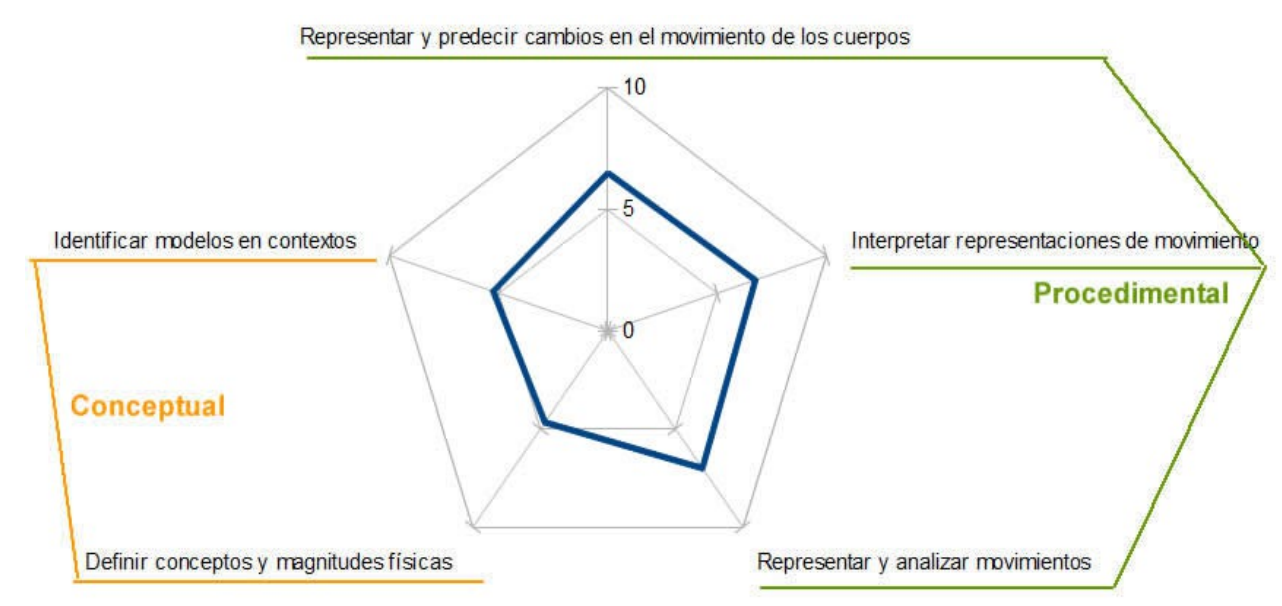

Figura 7. Valoración de las calificaciones medias del alumnado en preguntas evaluando aspectos de las dimensiones procedimental y conceptual.

Se han detectado algunos errores frecuentes, como la confusión entre velocidad y aceleración. «Como la aceleración del objeto 1 es mucho mayor que el 2, este sale más rápido de lo que iba y el uno ha perdido velocidad», la confusión entre peso y masa «El segundo choca más fuerte porque pesa más» o la confusión entre masa y volumen «Cuando más grande es el objeto más fuerza hay que hacer para moverlo».

\section{Análisis de los resultados de la encuesta}

La encuesta al alumnado (figura 8) muestra que los alumnos consideran que la actividad ha desarrollado aspectos conceptuales, procedimentales y epistémicos, en particular la representación e interpretación de gráficos y el carácter socio-científico de las decisiones. En cambio el trabajo con fuerzas y la conservación del movimiento son apercibidos como elementos menos aprendidos por el alumnado. En conversaciones con el alumnado destacan que han aprendido a no fiarse de las apariencias y esperar a analizar las evidencias antes de tomar una decisión.

Se produce una evolución interesante a nivel epistemológico en las concepciones de los alumnos sobre el trabajo de los peritos. Se incrementa la proporción de alumnos que considera el trabajo de los peritos una tarea científica y disminuye la proporción de alumnos que considera que las conclusiones son seguras. En conversaciones con el alumnado se deduce que inicialmente identificaban "científico» con la necesidad de realizar una prueba química en un laboratorio y no como un contraste de datos con modelos. Así mismo, la evolución indica que muchos alumnos dejan de considerar que lo científico tenga que ser seguro, algo epistemológicamente positivo. En lo que respecta a las herramientas didácticas, el alumnado mayoritariamente valora como útiles todas las herramientas, especialmente el hecho de tener un guión que ordenara las etapas del proyecto, y el trabajo con representación gráfica y manipulación de objetos. En cambio, son menos los que valoran como útiles el modelo Frayer o el trabajo realizado en el estudio de movimientos en el patio. Si bien de forma general los alumnos se han implicado en la actividad y las discusiones conceptuales y epistémicas que ésta ha generado, algunos equipos de peritos han mostrado una tendencia a la dispersión que ha necesitado ser reconducida y ha impactado en los resultados del grupo. 
a)

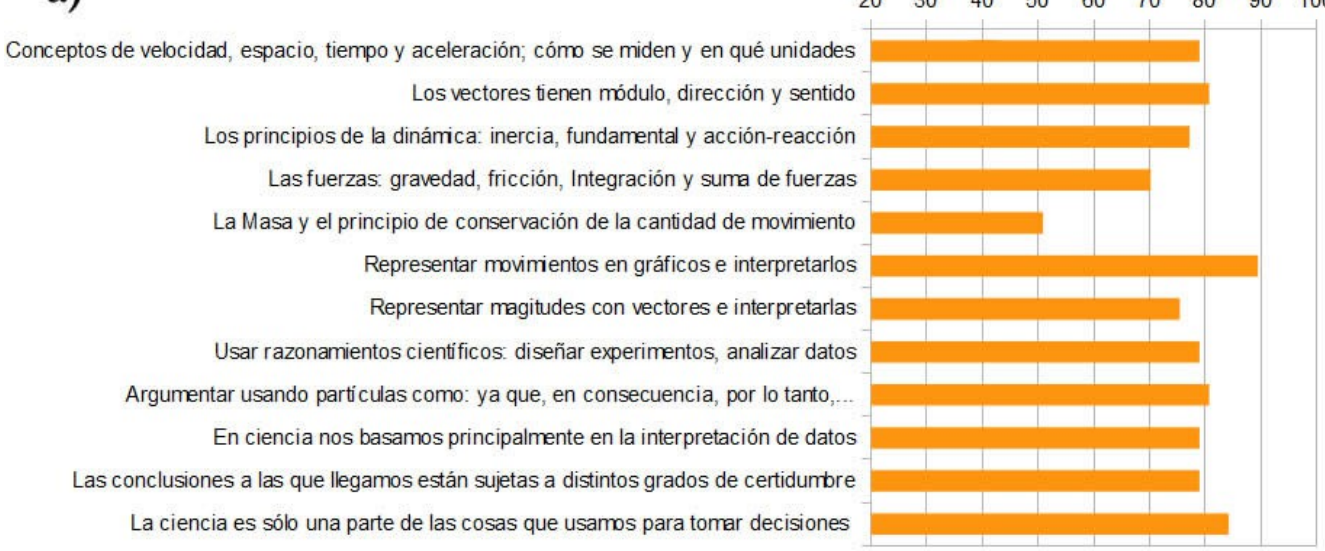

b)

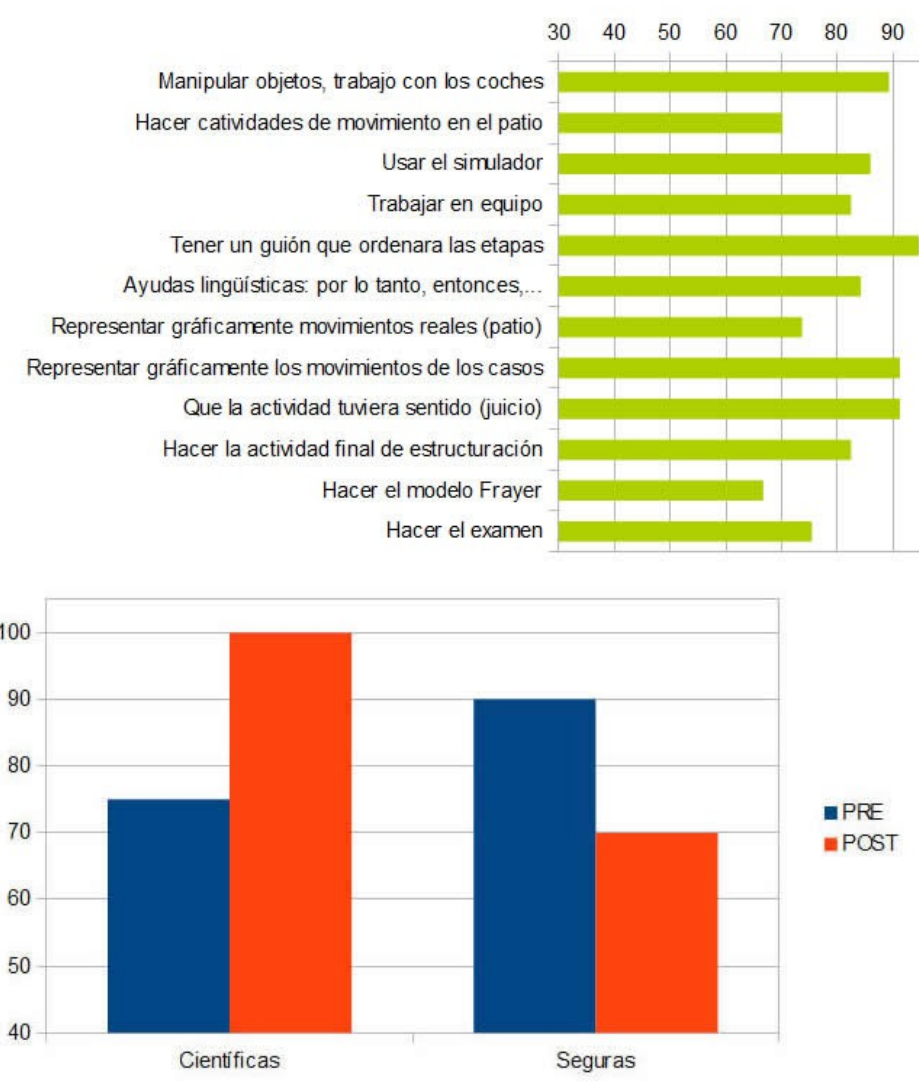

Figura 8. Porcentaje de alumnos que a) consideran que han aprendido cada ítem; b) consideran que una estrategia o herramienta didáctica les ha sido útil. C) evalúan como «científicos» o «Seguras» las conclusiones de los peritos.

\section{Discusión y conclusiones}

Los estudios de caso como metodología para el desarrollo de la competencia científica

La actividad propuesta ofrece espacios para el desarrollo de las tres dimensiones de la competencia científica tal como se representa en la roseta de dimensiones de la figura 9. Por ejemplo, la participación del alumnado en la vista del juicio ha sido un buen ejercicio epistémico, pues es un contexto en el que de forma natural se requiere determinar las fuentes de las conclusiones y el grado de certidumbre de las conclusiones. 
El examen realizado a los alumnos muestra que los alumnos desarrollan aspectos conceptuales y procedimentales, especialmente estos últimos (representación e interpretación de gráficos, diseño de experimentos...). Desde un punto de vista conceptual, en el análisis de los accidentes los alumnos dejan de apelar únicamente a aspectos como la atención y pericia para usar principios y magnitudes físicas (inercia, masa, fricción...). La dinámica indagadora ha permitido que el alumnado desarrolle de forma propia conceptos necesarios («punto de referencia») y relaciones de magnitudes («A más fricción, mayor desaceleración»).

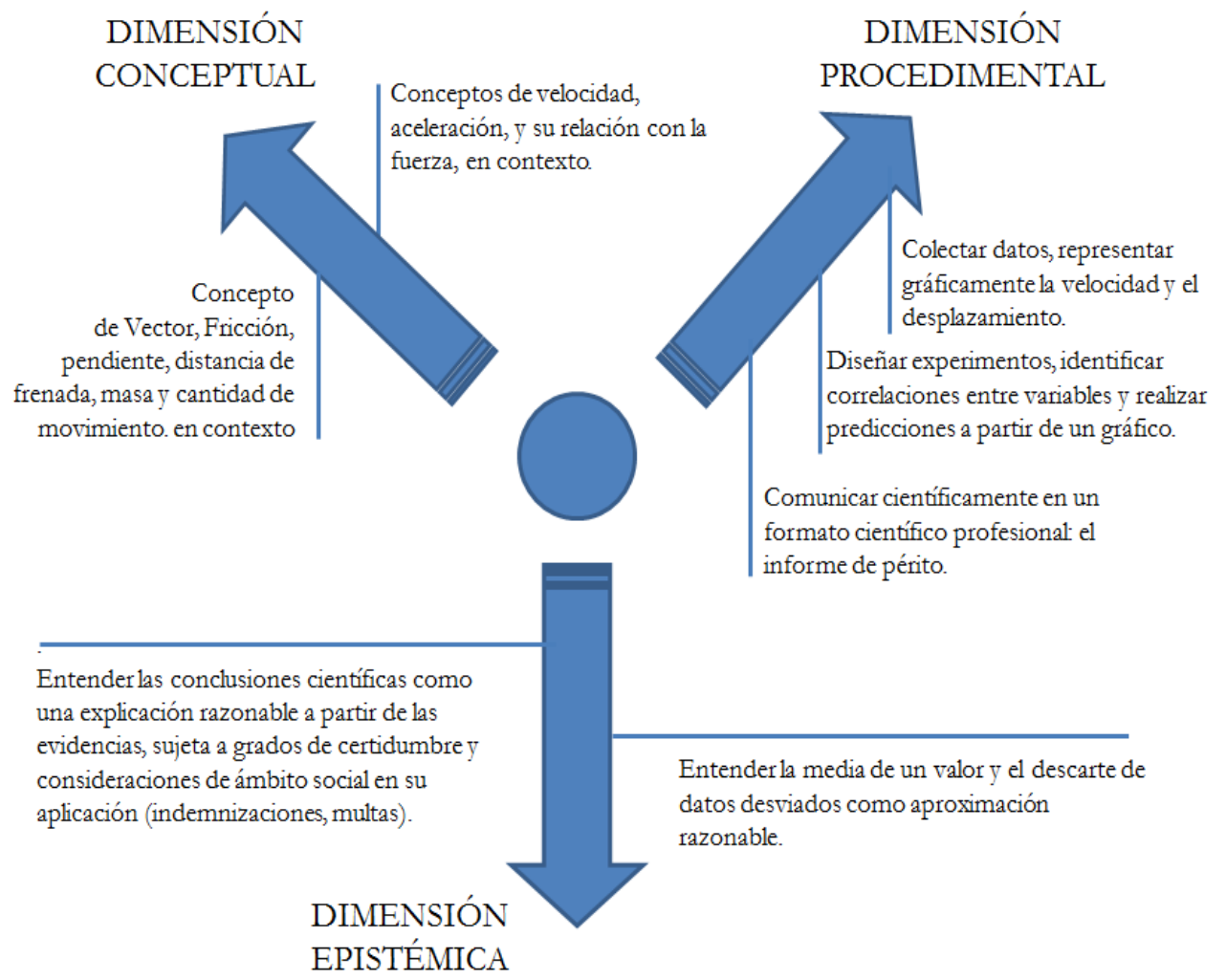

Figura 9. Roseta de dimensiones elaborada para el análisis de las aportaciones a la competencia científica que ofrece la actividad, elaborada como se propone en Domènech-Casal (2017a).

También los aspectos epistémicos son bien valorados por el alumnado: los ítems epistemológicos de la encuesta (en ciencia nos basamos en la interpretación de datos, las conclusiones están sujetas a grados de certidumbre, la ciencia es sólo una parte de lo que usamos para tomar decisiones) reciben apoyo de alrededor del $90 \%$. El cambio producido entre el inicio y el final de la actividad (incremento del número de alumnos que consideran las conclusiones del peritaje como científicas y disminución del número de alumnos que las consideran seguras) parece indicar un cambio de visión de lo que es la ciencia (un proceso argumentativo y no un análisis de laboratorio) y de su base epistemológica (las conclusiones no son seguras, sino que existen grados de certidumbre).

\section{Las concepciones del alumnado sobre cinemática y dinámica}

A lo largo de la secuencia y en los diálogos en el aula se han detectado varias concepciones alternativas, ya detectadas previamente en la literatura. En particular, se han detectado confusiones entre masa y peso (si bien parecen deberse más a un uso indebido de léxico que a una confusión conceptual). También -en el trabajo con la representación de los accidentes mediante vectores- algunos alumnos han mostrado dificultades para determinar a qué magnitud hacían referencia los vectores -aceleración, velocidad, o fuerza- que en la 
conversación resumen de forma genérica como «impulso», un término que los alumnos han asociado a menudo con el significado de inercia.

Los alumnos no han presentado dificultades en identificar la aceleración como negativa en la desaceleración o la velocidad como negativa en el retroceso, algo que suponemos se debe al trabajo intensivo y contextual con gráficos de representación de movimiento.

En las conversaciones con los alumnos y las justificaciones de la pregunta 2 del examen, se pone en evidencia que los alumnos adquieren una versión rudimentaria del principio de conservación de la cantidad de movimiento que podría resumirse en: 1) al chocar entre ellos, los cuerpos «se reparten» la velocidad; 2) este proceso tiende a equilibrar la cantidad de movimiento de las dos partículas. Esta concepción errónea de cantidad de movimiento no considera los dos cuerpos como un solo sistema ni que la cantidad de movimiento se conserva para todo el sistema.

\section{Lecciones de la experiencia para el diseño de actividades de estudio de caso}

La actividad se ha construido siguiendo las pautas propuestas anteriormente en otros casos de diseño de actividades (Domènech-Casal 2017a): elección de escenario y pruebas, secuenciación de etapas y productos, y análisis de aportaciones a las dimensiones de la competencia científica.

El hecho de ubicar los casos como punto inicial del problema, incluso antes de introducir ningún concepto ha generado una dinámica indagativa interesante, en especial en la etapa 3 , en la que los alumnos determinan, primero intuitivamente y después mediante experimentos, las relaciones entre magnitudes físicas con el objetivo de resolver un problema.

Si bien el nivel de desarrollo de la dimensión conceptual es suficiente para este nivel educativo, observamos la necesidad de apoyos didácticos que ayuden al alumnado a sistematizar y establecer relaciones entre conceptos más allá de su aplicación para la resolución de un problema. En lo referente a las herramientas didácticas, las dificultades del alumnado en la dimensión conceptual se han intentado subsanar mediante el uso de discusiones en el aula y el modelo Frayer. Los alumnos valoran de forma poco positiva esta herramienta, y se han obtenido pocos resultados, y consideramos que en futuros diseños de actividades ésta debe completarse con organizadores gráficos más globales como mapas conceptuales.

La actividad permite desarrollos ulteriores en varios sentidos:

aIncorporando en los vídeos producidos por el alumnado una etapa de edición con técnicas de mash-up que permitan superponer en el mismo vídeo los vectores u otras representaciones (trayectorias, ángulos) tal como proponen otros autores (Ezquerra, Iturrioz, Díaz 2012).

aVinculando la temática a controversias socio-científicas del contexto de la seguridad vial como la obligatoriedad de normas de protección (cinturón de seguridad y casco, límites de velocidad, pruebas médicas en conductores), las multas y penas por conducción temeraria, etc.

aEn cursos superiores, incorporando el cálculo vectorial y la trigonometría como herramienta cuantitativa.

El lector encontrará de interés otras secuencias de estudios de caso (Domènech-Casal 2017a) elaboradas en el marco del itinerario ProyectandoBioGeo ${ }^{3}$ del proyecto $\mathrm{C}^{4}{ }^{4}$ (Domènech-Casal

\footnotetext{
$\overline{3}$ Itinerario de Aprendizaje Basado en Proyectos para Biología y Geología 4 ESO (https:/ /sites.google.com/site/ proyectandobiogeo/).

4 Proyecto C3 para la Creación del Conocimiento Científico en el aula (https://sites.google.com/a/xtec.cat/c3/ home).
} 
2016, 2017b) y la colección de secuencias de estudio de caso que ofrece la Universidad de Buffalo para distintos niveles educativos (http://sciencecases.lib.buffalo.edu/cs/).

\section{Agradecimientos}

Reflexiones incluidas en este artículo se enmarcan en la investigación metodológica del grupo de investigación consolidado LICEC (referencia 2014SGR1492) por AGAUR y financiado por el Ministerio de Economía y Competitividad (referencia EDU2015-66643-C2-1-P). El Proyecto CRASH es el desarrollo de una idea original co-creada en el encuentro \#betacamp16 por el nodo de profesorado \#TauronsIndagadors http://www.betacamp.cat/taurons-indagadors/ y ulteriormente desarrollada por profesorado del grupo de trabajo EduWikiLab https://eduwikilab.wordpress.com/. Los autores agradecen la participación a los profesores y a los coordinadores de ambos eventos la generación de espacios de cooperación.

\section{Referencias}

Alzugaray G. E., Enrique C. M., Esterkin C. R. (2014) Conceptos y preconceptos de cinemática y dinámica en ingresantes a carreras de ingeniería. Latin American Journal of Physics Education 8 (1), 31-37.

Blanco A., España E., Rodríguez F. (2012) Contexto y enseñanza de la competencia científica. Alambique. Didáctica de las Ciencias Experimentales 70, 9-18.

Chiva O., Martí M. (2016) Métodos pedagógicos activos y globalitzadores. Conceptualización y propuestas de aplicación. Barcelona: Graó.

Christensen C. R., Hansen A. J. (1986) Teaching and the Case Method. Boston: Harvard Business School Publishing Division.

Cliff W. H., Wright A. W. (1996) Directed case study method for teaching human anatomy and physiology. Advances in Physiology Education 15, 19-28.

Cliff W. H., Nesbitt-Curtin L. (2000) The directed case method. Journal of College Science Teaching 30 (1), 64-66.

Couso D. (2014) De la moda de «aprender indagando» a la indagación para modelizar: una reflexión crítica. XXVI Encuentro de Didáctica de las Ciencias Experimentales. Huelva.

del Moral S. (2016) Del conocimiento al aprendizaje, un camino sin retorno. Materias y competencias en el ABP. Cuadernos de Pedagogía 472, 51-54.

Domènech-Casal J. (2015) Una secuencia didáctica de modelización, indagación y creación del conocimiento científico en torno a la deriva continental y la tectónica de placas. Revista Eureka sobre Enseñanza y Divulgación de las Ciencias 12 (1), 186-197.

Domènech-Casal J. (2016) Proyecto C3: indagación científica, lengua y contextos en la ESO. Aula de Secundaria 19, 15-19.

Domènech-Casal J. (2017a) Aprendizaje Basado en Proyectos y Competencia Científica. Experiencias y propuestas para el método de Estudios de Caso. X Congreso Enseñanza de las Ciencias Septiembre 2017 (número extraordinario), 5177-5183.

Domènech-Casal J. (2017b) Proyectando BioGeo, un itinerario de trabajo por proyectos contextualizados basado en la indagación y la Naturaleza de la Ciencia. Alambique. Didáctica de las Ciencias Experimentales 89, 54-61.

Domènech-Casal J. (en edición) Contexto y modelo en el Aprendizaje Basado en Proyectos. Apuntes para el ámbito científico. Alambique. Didáctica de las Ciencias Experimentales. 
Ezquerra A., Iturrioz I., Díaz M. (2012) Análisis experimental de magnitudes físicas a través de vídeos y su aplicación al aula. Revista Eureka sobre Enseñanza y Divulgación de las Ciencias 9 (2), 252-264.

Frayer D., Frederick W. C., Klausmeier H. J. (1969) A Schema for Testing the Level of Cognitive Mastery. Madison, WI: Wisconsin Center for Education Research.

Fuentes C. (2016) Preconceptos de cinemática y fuerza en estudiantes que inician sus estudios de ingeniería. Revista Electrónica de Enseñanza de las Ciencias 15 (1), 43-52.

Garrido A., Simarro C. (2014) El nou marc d'avaluació de la competència científica PISA 2015: Revisió i reflexions didàctiques. Revista Ciències 28, 21-26.

Gilbert J. K. (2006) On the nature of "context" in chemical education. International Journal of Science Education 28 (9), 957-976.

Glew R. H. (2003) The problem with problem-based medical school education: Promises not kept. Biochemistry and Molecular Biology Education 31, 52-56.

Guidugli S., Fernández C., Benegas J. (2004) Aprendizaje activo de la cinemática lineal y su representación gráfica en la escuela secundaria. Enseñanz̧a de las Ciencias 22 (3), 463-472.

Guillaumes M. (2016) Cuando aprender es un reto. Cuadernos de Pedagogía 472, 55-58.

Hattie J. (2009) Visible Learning: A synthesis of over 800 meta-analyses relating to achievement. Routledge: New York.

Herreid C. F. (1994) Case studies in science: A novel method for science education. Journal of College Science Teaching 23 (4), 221-229.

Herreid C. F. (2003) The death of problem-based learning? Journal of College Science Teaching 32 (6), 364-66.

Hewson P. W., Beeth M. E. (1995) Enseñanza para un cambio conceptual: ejemplos de fuerza y de movimiento. Enseñanza de las Ciencias 13 (1), 25-35.

Hudson J. N., Buckley P. (2004) An evaluation of case-based teaching: Evidence for continuing benefit and realization of aims. Advances in Physiological Education 28, 15-22.

Hurovich V. L., Azpiazu S., Cucci G., Joselevich M. (2015) Hacia la integración de las TIC en el aula: una propuesta de trabajo sobre cinemática utilizando sensores electrónicos de distancia. Revista de Enseñanza de la Física 27, 525-531.

Hutchings P. (1993) Using Cases to improve college teaching: a guide to a more reflective practice. Washington, DC: American Association for Higher Education.

Jiménez-Aleixandre M. P. (2011) 10 Ideas Clave. Competencias en argumentación y uso de pruebas. Barcelona: Graó.

Kilpatrick W. E. (1918) The Project Method: the use of the purposeful act in the educative process. New York: Teachers College, Columbia University.

Larmer J., Mergendoller J., Boss S. (2015) Setting the Standard for Project Based Learning: A Proven Approach to Rigorous Classroom Instruction. Alexandria: ASCD.

Llewellyn D. (2005) Teaching High School Science through Inquiry: A case study approach. Thousand Oaks, CA: Corwin Press \& NSTA Press.

López V. (2015) L'acceleròmetre del mòbil. Una alternativa al sensor de distància? Recursos de Física 15, 5-8. 
MECD (2015) Ministerio de Educación, Cultura y Deporte. Real Decreto 1105/2014, de 26 de diciembre, por el que se establece el currículo básico de la Educación Secundaria Obligatoria y del Bachillerato. Boletín Oficial del Estado 2015 (3), I, 169.

Mendoza A. J., Ripoll L., Ruz L. (2005) Instrumentos para la enseñanza - aprendizaje de los vectores en cinemática. Educación y Pedagogía 17 (43), 93-107.

Miró M., Saperas A. M., Tarragó M., Tordera M. R., Domènech-Casal J. (2016) Cinc experiències i reflexions metodològiques sobre l'Aprenentatge Basat en Projectes a les Ciències. Revista Ciències 32, 23-33.

Mora C., Herrera D. (2009) Una revisión sobre ideas previas del concepto de fuerza. Latin American Journal of Physics Education 3 (1), 72-86.

OCDE (2013) PISA 2015. Draft Science Framework.

Osborne J., Dillon J. (2008) Science Education in Europe: Critical Reflections. Londres: Nuffield Foundation.

Picquart M. (2008) ¿Qué podemos hacer para lograr un aprendizaje significativo de la física? Latin American Journal of Pbysics Education 2 (1), 29-36.

Pollatsek H., Schwartz R. (1990). Case studies in quantitative reasoning: An interdisciplinary course. Extended Syllabi of the New Liberal Arts Program. New York: SUNY/Stony Brook.

Rocard Y. (2007) Science Education Now. Report EU22-845, EU, Brussels.

Sánchez J. L., Oliva J. M., Rosado L., Cruz M. I. (1993) Detección de las ideas previas en cinemática utilizando la composición de movimientos. Investigación en la Escuela 19, 105116.

Sanmartí N. (2016) Trabajo por proyectos: ¿Filosofía o metodología? Cuadernos de Pedagogía $472,44-46$.

Sanmartí N., Márquez C. (2017) Aprendizaje de las ciencias basado en proyectos: del contexto a la acción. Ápice, Revista de Educación Científica 1 (1), 3-16.

Sebastià J. M. (1984) Fuerza y Movimiento, la interpretación de los estudiantes. Enseñanz̧a de las Ciencias 2 (3), 161-169.

Sebastià J. M. (1988) Algunos patrones de interpretación espontánea frente a situaciones de dinámica clásica. Revista de Enseñanza de la Física 2 (2), 80-88.

Sebastiany A. P., Pizzato M. C., Diehl I. V., Miskinis T. D. (2013) Aprendiendo a investigar por medio de la ciencia forense e investigación criminal. Revista Eureka sobre Enseñanza y Divulgación de las Ciencias 10 (3), 480-490.

Simarro C., Couso D., Pintó R. (2013) Indagació basada en la modelització: un marc per al treball pràctic. Revista Ciències 25, 35-43.

Simarro C., López V., Cornellà P., Peracaula M., Niell M., Estebanell M. (2016) Més enllà de la programació i la robòtica educativa: el pensament computacional en l'ensenyament STEAM a infantil i primària. Revista Ciències 32, 38-46.

Viennot L. (2011) Els molts reptes d'un ensenyament de les Ciències basat en la indagació: ens aportaran múltiples beneficis en l'aprenentatge? Revista Ciències 18, 22-36.

Wasserman S. (1999) El estudio de casos como método de enseñanz̧a. Buenos Aires: Amorroutu Editores. 